\title{
Self-assembled, Fluorine-rich Porous Organic Polymers: A Class of Mechanically Stiff and Hydrophobic Materials
}

\author{
Soumya Mukherjee, ${ }^{[\mathrm{a}] \dagger}$ Zhixin Zeng,${ }^{[\mathrm{b}] \dagger}$ Mandar M. Shirolkar,,${ }^{[\mathrm{c}, \mathrm{d}]}$ Partha Samanta, ${ }^{[\mathrm{a}]}$ Abhijeet K. \\ Chaudhari, ${ }^{[b]}$ Jin-Chong Tan, ${ }^{*[b]}$ and Sujit K. Ghosh ${ }^{*[a, e]}$
}

\begin{abstract}
Fluorous organic building blocks have been cogently harnessed to develop two self-assembled, hydrophobic and fluorinated porous organic polymers (FPOPs) namely, FPOP-100 and FPOP-101. On comprehensive mechanical analyses of these functionalized triazine network polymers, they mark the introduction of mechanical stiffness among all porous organic network materials; recorded stiffness features analogous to their organic-inorganic hybrid polymer analogues, viz. metal-organic frameworks (MOFs). Further, the study presents a new paradigm for the simultaneous installation of mechanical stiffness and high surface hydrophobicity into polymeric organic networks, which has the utilitarian potential to be translated among all porous solids. Control experiments with nonfluorinated congeners could underline the key role of fluorine, in particular, bis(trifluoromethyl)-functionalization behind realizing the dual features of mechanical stiffness and superhydrophobicity.
\end{abstract}

\section{Introduction}

Last decade has observed a striking upsurge of exciting research findings in the realm of porous organic polymers (POPs), due to their remarkably high surface area, low density, feasibility of appending reactive functional moieties at the pore/channel surface, accompanied by a wide miscellany of framework compositions. ${ }^{[1]}$ A huge potential of application for such functionalized materials has been predominantly realised in the domains of molecular storage, ${ }^{[2]}$ heterogeneous catalysis, ${ }^{[3]}$ sensing of molecules/ metal-ions, ${ }^{[4]}$ selective gas/solvent adsorption, ${ }^{[5]}$ opto-electronics, ${ }^{[6]}$ drug delivery ${ }^{[7]}$ among many others. ${ }^{[8]}$ In this context, gaining coherent insights into the mechanical behaviour for this class of solid-state materials plays quite a key role to the rational design driven optimal performance of a multitude of technological applications envisaged thereafter. ${ }^{[2 \mathrm{~b}]}$

More recently, covalent organic frameworks (COFs) have emerged as a distinctive class of POPs, with extended porous ordered networks bearing open channels constituted from pre-

[a] Department of Chemistry, Indian Institute of Science Education and Research (IISER) Pune. Dr. Homi Bhabha Road, Pashan, Pune411008, India. E-mail: sghosh@iiserpune.ac.in

[b] Department of Engineering Science, University of Oxford, Parks Road, Oxford OX1 3PJ, UK. E-mail: jin-chong.tan@eng.ox.ac.uk

[c] Department of Physics, Tamkang University, Tamsui, 251, Taiwan

[d] Hefei National Laboratory for Physical Science at the Microscale, University of Science and Technology of China, Hefei, Anhui230026, People's Republic of China.

[e] Centre for Energy Science, IISER Pune, Pune-411008, India † S.M. and Z.Z. have contributed equally.

Supporting information for this article is given via a link at the end of the document. designed organic motifs. ${ }^{[9]}$ The tailored composition of these lightweight materials confer the unique combination of low mass density, permanent porosity as well as mechanical robustness. ${ }^{[10]}$ Such relatively underexplored porous organic polymers closely resemble the well-studied class of coordination polymers possessing tuneable porosity, viz. the metal-organic frameworks (MOFs) where the coordination bonds are replaced with strong covalent bonds, aimed at the unification of chemical and structural stability to yield permanent porosity. With a parallel but slightly underexplored progress, the field of nitrogen-rich covalent triazine-based frameworks (CTFs) has rapidly flourished to emerge as a vital subclass of covalent organic frameworks, ${ }^{[11]}$ thanks to their excellent thermal stability and chemical inertness. ${ }^{[12]}$ Facile acid-catalysed room-temperature synthesis protocols act as an added advantage to the present landscape of promising CTF materials. ${ }^{[13]}$ In the pursuit of incorporating diverse functionalities among CTFs, implementation of suitable material design principles is considered state-of-the-art, arguably in view of their intertwined structure-property relationship. ${ }^{[14]}$ For example, fluorines are well-regarded for imparting hydrophobicity to coordination nanospace and result into hydrophobic surface properties. ${ }^{[15]}$ However, influence of densely packed fluorines on the surface characteristics of COFs, and more precisely, CTFs remain unexplored. There are a few reports of mechanical stiffness studies on nanoporous MOFs: Isoreticular MOFs (IRMOFs) including MOF-5, HKUST-1 or the prototypal ZIFseries, ${ }^{[2 b, 16]}$ mechanical behaviour hitherto remain unexplored in organic network architectures, like CTFs.

Lack of crystallinity has largely precluded the access to obtain adequate structural information among the non-crystalline examples of covalent triazine network polymers, often denoted as CTF analogues regardless of their dwindled framework characteristic. ${ }^{[17]}$ Being amorphous, the latter class often remains elusive in terms of direct observation of structural traits, experimentally determined porosity serving as their primary footprint of characterization. ${ }^{[17]}$ By definition, porous framework materials like COFs and CTFs shall possess crystallinity. ${ }^{[18]}$ This allows classification of amorphous organic polymers with microporosity under the wider family of POPs, in sync with the chronological development. ${ }^{[19]}$ Traditionally, POPs are categorized among hyper-cross-linked polymers (HCPs), ${ }^{[20]}$ polymers of intrinsic microporosity (PIMs), ${ }^{[21]}$ COFs, CTFs, porous aromatic frameworks (PAFs), ${ }^{[22]}$ and conjugated microporous polymers (CMPs). ${ }^{[23]}$ This nomenclature enables diversely synthesized and functionalized organic network solids to be placed under an all- 
a)
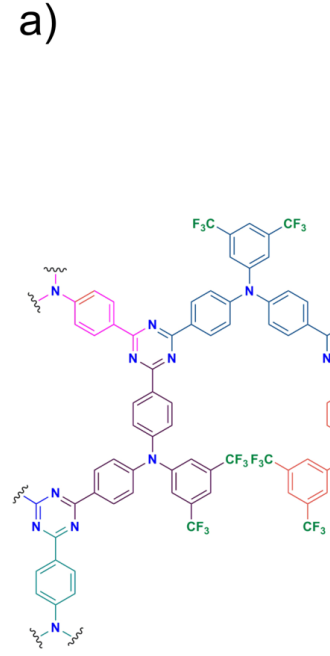
mechanically stiff monolithic compounds FPOP-100 and FPOP-101, respectively. b)

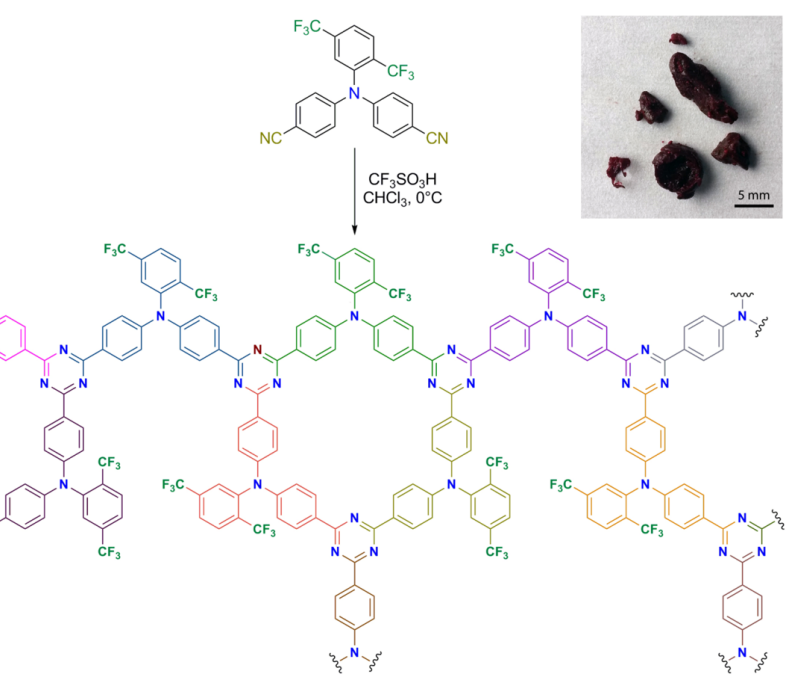

inclusive category of POPs, subject to upholding the criterion of porosity. ${ }^{[24]}$ In this report, a fluorine-rich pair of triazine network based POPs introduce the property of mechanical stiffness among organic network solids. Despite their close resemblance to CTFs, due to their identical building blocks, i.e. polynitrile synthetic components undergoing cyclotrimerisation reaction, absence of any long-range order delineates the currently reported materials as POPs. Two porous, triazine-based and fluorinated organic polymers viz. FPOP-100 and FPOP-101 (F denotes fluorinated), built from two bis(trifluoromethyl)(- $\left.-\mathrm{CF}_{3}\right)$-rich isomeric dinitriles (positional isomers) are presented in the current report. The two amorphous FPOPs, owing to surface functionalization of the constituent triazine networks by means of a priori introduction of $-\mathrm{CF}_{3}$ groups, results in improved hydrophobic properties. Although such highly hydrophobic traits have been realised in congener families of fluorinated porous crystalline networks like MOFs and imine-based COFs, ${ }^{[15,25]}$ pore surface engineering principles resulting in the demonstration of superhydrophobic properties among POPs i.e. organic porous network solids have not been reported yet. Meanwhile, mechanical properties, in particularly, Young's modulus $(E)$ and hardness $(H)$, are of crucial importance to the practical uses of materials. Among POPs, dense fluorine rich environment offers a unique prefunctionalization strategy, leading to mechanical stiffness properties, comparable to the stiffness found in other molecular porous materials, e.g. a few zeolites and MOFs. ${ }^{[2 b, 16,26]}$ Thermal and chemical robustness further widens the applicability of FPOPs under an ample range of operating conditions.

\section{Results and Discussion}

Following the room-temperature synthesis protocol of CTFs established by Cooper and co-workers, ${ }^{[13 a]}$ the family of fluorous organic porous triazine polymers studied herein, viz. FPOP-100 and FPOP-101 were prepared via acid-catalysed room temperature reactions of the respective dicyanomonomers ( $L$ ' and L") in $\mathrm{CHCl}_{3}$ as a solvent (Fig. 1). It was observed that both FPOPs were insoluble in a wide range of organic solvents (dimethylformamide (DMF), dimethylacetamide (DMAc), methanol, ethanol, tetrahydrofuran (THF), chloroform and hexane) and water. Despite extensive washing, their prominent insolubility revealed the robust nature of the FPOPs owing to the extended network formation coupled with the inherently stable triazine rings. ${ }^{[12 c, 27]}$ Preparation of the polymeric trimerised compounds were monitored from FT-IR spectra of the products (Fig. S24). IR spectra of the two FPOP materials revealed substantial differences when compared to the respective dinitrile precursors $L^{\prime}$ and $L^{\prime \prime}$, as the $C \equiv N$ stretching frequency for the monomers (at $2360 \mathrm{~cm}^{-1}\left(\mathrm{~L}^{\prime}\right)$, and $\left.2210 \mathrm{~cm}^{-1}\left(\mathrm{~L}^{\prime \prime}\right)\right)$ were absent in the resulting POPs, as shown in Fig. S24. Furthermore, characteristic peaks for the triazine ring at about $1520 \mathrm{~cm}^{-1}$ (FPOP-100) and $1500 \mathrm{~cm}^{-1}$ (FPOP-101) appeared, suggestive of cyclotrimerised functionality in each of them. Thermo-gravimetric analyses (TGA) under $\mathrm{N}_{2}$ atmosphere for the as-synthesized phases showed an initial $\sim 10 \%$ loss of guest solvent species occluded during the syntheses, followed by negligible weight loss until $\sim 300{ }^{\circ} \mathrm{C}$ (FPOP-100) and $\sim 315^{\circ} \mathrm{C}$ (FPOP-101) (Figures S18 and S19). Complementary thermoanalytical study was done using differential scanning calorimetry (DSC) (Fig. S34). Typical guest exchange protocol by lower boiling solvents (chloroform and THF, binary mixture (1:1)) followed by evacuation under vacuum resulted in complete desolvation of the compounds, since both the ensuing guest-free POP phases were found to suffer no weight loss till $\sim 300^{\circ} \mathrm{C}$. On the contrary, thermal stabilities for the corresponding monomer building blocks' (L' and L") were relatively poor (Figures S18 and S19), prototypal for such organic dinitrile monomers.

PXRD patterns for both the FPOPs (as-synthesized and the guest-free phases) show amorphous nature (Figure 2d). However, the broad diffraction peaks observed at $2 \theta=18.4^{\circ}$ and $19.5^{\circ}$ for FPOP-100 and FPO-101 respectively, suggested the existence of 
graphitic 2D layers. Such broad diffraction patterns hinder precise structure elucidation due to absence of any long-range ordering in the FPOP bulk samples. However, weak crystalline nature is often observed in POPs, especially in triazine network polymers prepared by room-temperature method. ${ }^{[13]}$ In fact, this suggests that our glassy polymer materials may comprise of non-coplanar, two-dimensional (2D) organic layers i.e. aromatic networks beyond any repeatable pattern, resembling hard carbon. ${ }^{[28]}$ Low temperature $(195 \mathrm{~K}) \mathrm{CO}_{2}$ adsorption isotherms for FPOP-100 and FPOP-101 afford BET surface areas of $261 \mathrm{~m}^{2} \mathrm{~g}^{-1}$ and 274 $\mathrm{m}^{2} \mathrm{~g}^{-1}$, respectively; corresponding to pore volumes of $\sim 0.1 \mathrm{~cm}^{3} \mathrm{~g}$ ${ }^{1}$ for both. The respective saturation uptake capacities were 92 $\mathrm{cm}^{3} \mathrm{~g}^{-1}$ and $94 \mathrm{~cm}^{3} \mathrm{~g}^{-1}$ (Figures 2a and $2 \mathrm{~b}$ ), implying extrinsic porosity. $\mathrm{N}_{2}$ and $\mathrm{H}_{2}$ adsorption isotherms recorded at $77 \mathrm{~K}$ exhibit low uptakes of $27 \mathrm{~cm}^{3} \mathrm{~g}^{-1}$ and $6 \mathrm{~cm}^{3} \mathrm{~g}^{-1}\left(\mathrm{~N}_{2}\right) ; 43 \mathrm{~cm}^{3} \mathrm{~g}^{-1}$ and $41 \mathrm{~cm}^{3} \mathrm{~g}$ ${ }^{1}\left(\mathrm{H}_{2}\right)$ for FPOP-100 and FPOP-101, respectively (Figures 2a and $2 b) . \mathrm{CO}_{2}$ isotherm derived pore size distribution analyses, i.e. Horvath-Kawazoe plots for both polymers showed ultramicropores $(<7 \AA)$ of uniform width $(\sim 5 \AA)$ (Figure 2c). Such low porosity signatures are in accord with their observed amorphous nature, both as a consequence of irregular packing among the constituent polymeric triazine networks. However, more importantly, the physisorption experiments conclude the porosity aspect for the POPs.

a)
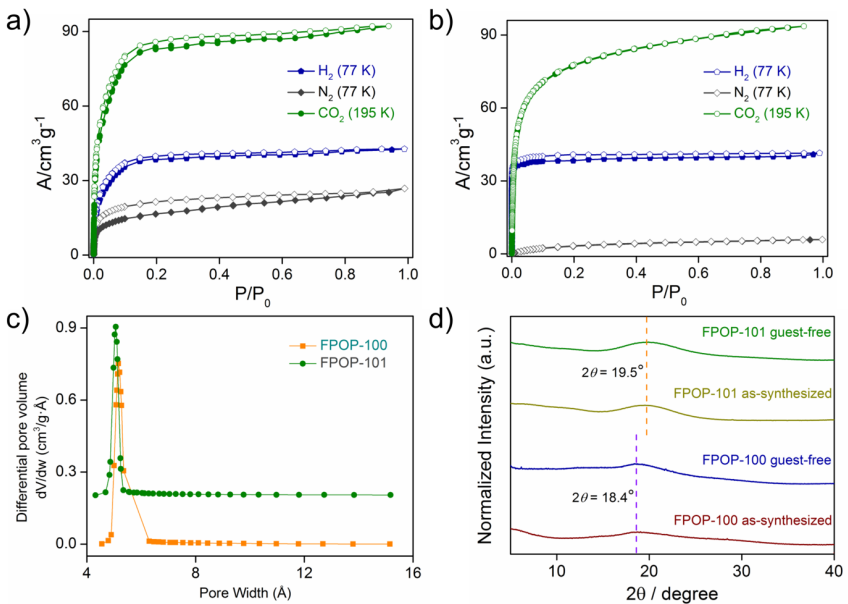

d)

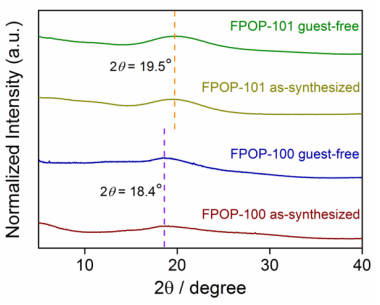

Figure 2. (a, b) Low temperature $\mathrm{CO}_{2}, \mathrm{~N}_{2}$ and $\mathrm{H}_{2}$ adsorption isotherms for FPOP-100 and FPOP-101, filled and empty symbols represent adsorption and desorption respectively; c) Horvath-Kawazoe (H-K) pore size distribution profiles for the FPOP pair, calculated from $\mathrm{CO}_{2}$ isotherms; d) Powder X-ray diffraction (PXRD) patterns of as-synthesized and guest-free phases of FPOPs: the broad diffraction peaks at $2 \theta=18.4^{\circ}$ (FPOP-100) and $19.5^{\circ}$ (FPOP-101) points to the existence of $2 \mathrm{D}$ layers in the polymers.

Solid-state Cross-Polarization Magic Angle Spinning Carbon-13 Nuclear Magnetic Resonance $\left({ }^{13} \mathrm{C}\right.$ CP-MAS SSNMR) spectra discloses characteristic peak for the triazine ring carbon atoms at $\sim 174 \mathrm{ppm}$ and other peaks between $\delta=100-240 \mathrm{ppm}$, which corresponds to the different aromatic carbon atoms as labelled in Fig. S28; 6 types of carbons for FPOP-100 [b-g], and 7 types for FPOP-101 [b-h]). The field emission scanning electron microscopy (FE-SEM) images show the stiff FPOP samples to be dense aggregations of particles forming similar homogeneous nanospheres (Figures S20 and S21 in the Supporting Information). Similar homogeneous nanosphere morphology is also revealed from the atomic force microscopy (AFM) images (3D as well as, roughness) of the triazine-based polymeric networks as shown in Figures S22 and S23. AFM morphology analyses also reveals that the average diameters for FPOP-100 and FPOP-101 nanospheres fall in very close range $(330 \mathrm{~nm}$ and $240 \mathrm{~nm}$, respectively); thus revealing comparable physical traits for the two FPOPs, possibly traceable to their isomeric precursors. Comparative analysis of the collected Raman spectra for the dinitrile monomers along with the two FPOPs exhibited the disappearance of $\mathrm{C} \equiv \mathrm{N}$ stretching bands $v(\mathrm{C} \equiv \mathrm{N}) \sim 2,220-2,230$ $\mathrm{cm}^{-1}$ (Fig. S27); simultaneously disclosing the similarity of network architectures for FPOP-100 and 101 species, evidenced by the similarity between their corresponding rotational-vibrational spectral signatures.

Fluorinated porous networks such as, fluorinated MOFs have been recognized in the recent literature to unveil stern water repellence features, alongside establishing their affinity toward hydrocarbon vapours like, benzene. ${ }^{[15,25 a, 29]}$ As an evidence of the fluorine-dense nature, water vapour sorption isotherm experiments with the FPOPs showed negligible uptake, even under saturation vapour pressure of water $(3.17 \mathrm{kPa}$ at $298 \mathrm{~K})$, while hydrophobic oil-constituent hydrocarbon benzene recorded slow opening assisted high saturation uptake-marked profiles (Figures $3 a$ and $3 b$ ). The stepwise nature of these benzene sorption isotherms suggest enhanced host-guest interactions with more and more surface-accumulation of the vapour sorbate benzene. In the case of densely crosslinked polymers like these, bearing very narrow openings (as suggested by the gas sorption isotherms), such hydrocarbon vapour sorption isotherm suggests the possible role of confinement effect- assisted slow filling of the fluorous voids. ${ }^{[15 a, 30]}$ Moreover, the contrasting vapour sorption isotherms (water and $\mathrm{C}_{6}$-hydrocarbon benzene) corroborates with the anticipated hydrophobicity of the FPOP materials. As a followup experiment, the surface wetting properties of the bulk amorphous phases were assessed by water contact angle (WCA) measurements. These experiments reproducibly registered static water contact angles as high as $159^{\circ}$ and $137^{\circ}$ for FPOP-100 and FPOP-101 respectively (with sessile drops), which evidenced the highly hydrophobic nature for both; FPOP-100 even notching superhydrophobicity. In fact, the aforesaid high WCA values mark these two POPs among only a few hydrophobic porous organic polymers, hitherto reported. ${ }^{[25 b, 31]}$ 

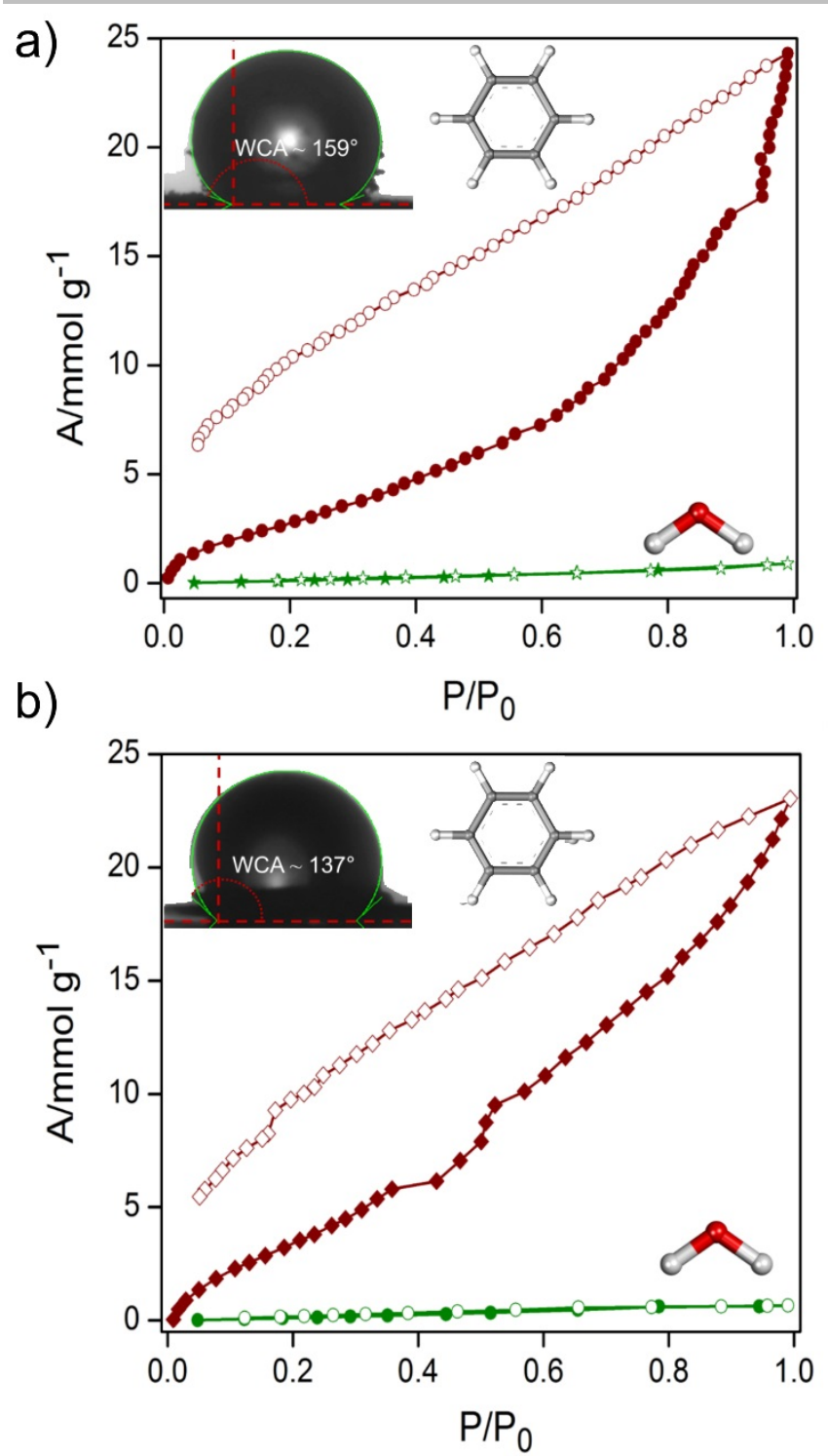

Figure 3. Contrasting water (green) and benzene (wine) vapour sorption isotherms for a) FPOP-100; b) FPOP-101, respectively. Insets for a) and b): Images of water drops slowly cast on the water-repellent surfaces of FPOP-100 and FPOP-101 pellets with superhydrophobic and highly hydrophobic WCAs ca. $159^{\circ}$ and $137^{\circ}$ respectively.

Using instrumented nanoindentation, we found that Young's modulus $(E)$ and hardness $(H)$ of FPOP-100 are $3.4 \pm 0.2 \mathrm{GPa}$ (assuming that the Poisson's ratio is 0.3 ) and $230 \pm 20 \mathrm{MPa}$, respectively. By contrast, $E$ and $H$ of FPOP-101 are $3.3 \pm 0.3 \mathrm{GPa}$ and $290 \pm 40 \mathrm{MPa}$, respectively. FPOP-100 with horizontal bis(trifluoromethyl) (-CF $)$ groups is hereby of comparable level of stiffness, but present considerably lower hardness relative to FPOP-101 that possesses diagonal $-\mathrm{CF}_{3}$ groups. A plausible explanation for the lower stiffness of FPOP-101 is the wider angle between $-\mathrm{CF}_{3}$ groups, as this might affect the network packing. In addition, under identical loading conditions, FPOP-100 exhibits less creep-induced deformation than FPOP-101 (Fig. 4b). Scattering in the results of $E$ (Fig. 4c) and $H$ (Fig. 4d) is most likely
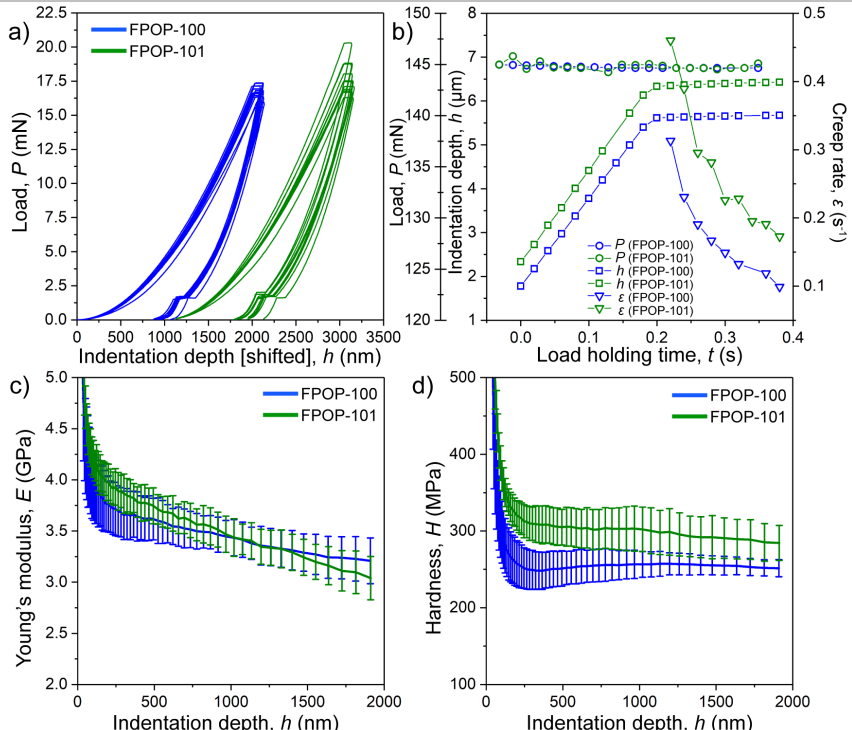

Figure 4. MTS nanoindentation on FPOPs: a) load vs. deformation curves; b) creep tests by applying the cyclic impact (Fig. S33); c) Young's modulus and d) hardness vs. indentation depth.

due to microstructural inhomogeneity, viz. non-uniformly distributed porosity. At shallow indentation of under $\sim 250 \mathrm{~nm}$ in

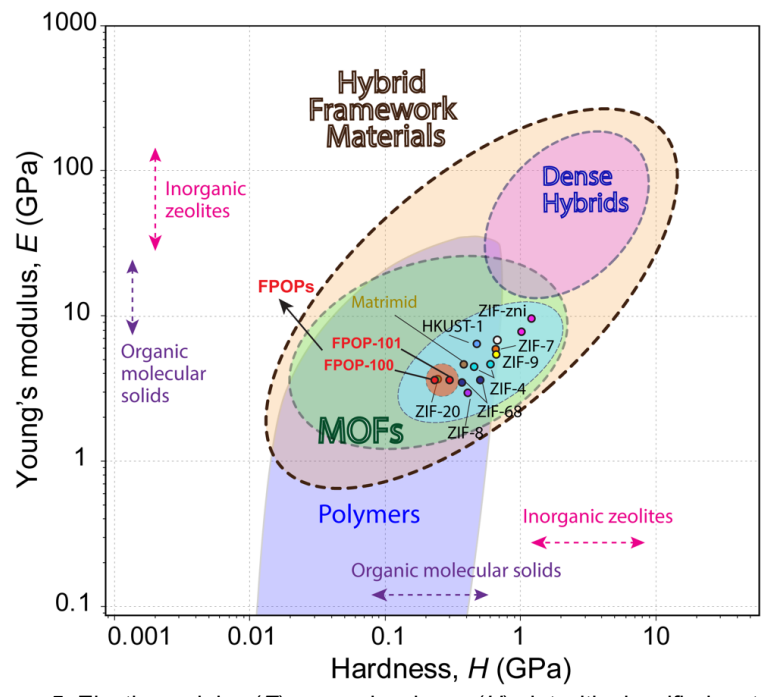

Figure 5. Elastic modulus $(E)$ versus hardness $(H)$ plot with classified materials property map, ${ }^{[2 b]} E$ and $H$ of FPOP-100 and FPOP-101 approximate to MOFs such as ZIFs, and resemble glassy polymers, one of which is matrimid.

Table 1. Energy dissipation at the first impact cycle (assuming the volume is the Berkovich indenter volume under the sample surface at maximum depth)

\begin{tabular}{ccc}
\hline & FPOP-100 & FPOP-101 \\
\hline $\begin{array}{c}\text { Energy lost per } \\
\text { volume } \\
\left(\times 10^{8} \mathrm{~J} / \mathrm{m}^{3}\right)\end{array}$ & 4.59 & 3.48 \\
$\begin{array}{c}\text { Energy lost per } \\
\text { mass }(\mathrm{J} / \mathrm{g})\end{array}$ & 0.32 & \\
\hline
\end{tabular}

this study, $E$ and $H$ are overestimated because the Berkovich indenter is not perfectly sharp, and thus at the tip apex, the actual contact area is larger than in ideal assumption. Therefore, $E$ and $H$ values were determined by averaging the values after indenting over $500 \mathrm{~nm}$. 
Many factors could interfere with the accurate measurement of contact area (such as subsurface cracking) and influence the recovery of the material resulting in a distorted unloading curve (such as creep effect[17b]). Inspection of Fig. $4 d$ shows that $H$ is unaffected by increasing indentation depth, which suggests no appreciable subsurface cracks are generated after indenting down to $2 \mu \mathrm{m}$. The descending trend of $E$ could be attributed to microstructural inhomogeneities of FPOP-100 and -101. Under the optical microscope, FPOP-101 sample shows more non-uniformly distributed micropores than FPOP-100, and this gets further reflected in the higher $\mathrm{N}_{2}$ uptakes as well as the higher creep rate (Fig. 4b) of FPOP-101.

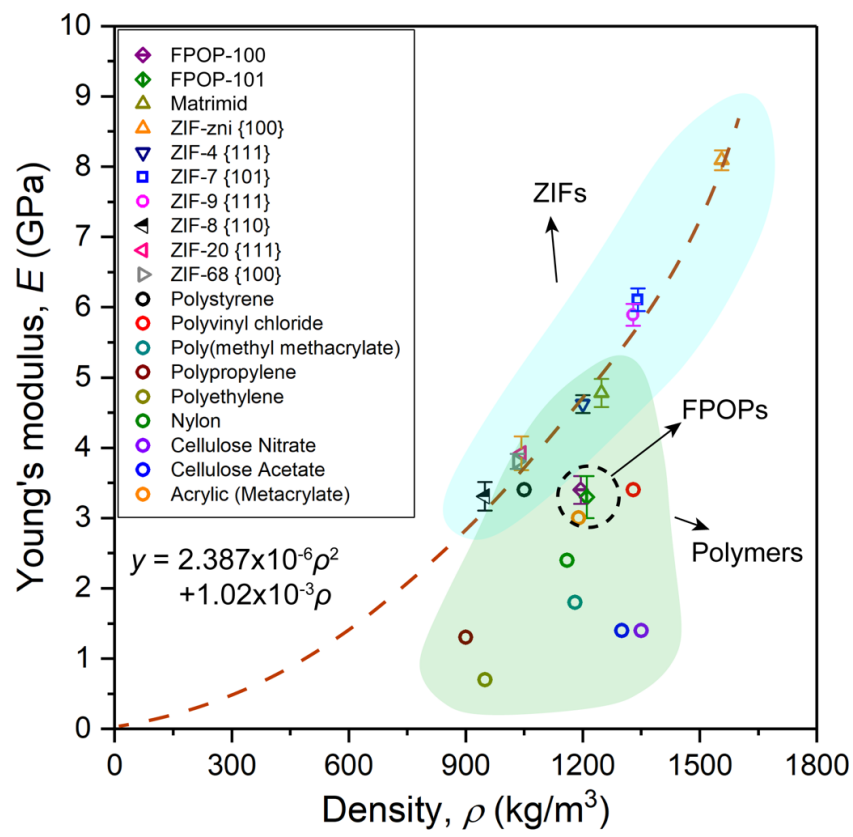

Figure 6. Young's modulus $(E)$ versus density $(\rho)$ plot showing the correlation between $E$ and $\rho$ for FPOPs, ZIFs, and ten common polymers. ${ }^{[2 b]}$

As shown in the materials selection map (Fig. 5), ${ }^{[2 b, 26]}$ Young's moduli and hardness of FPOP-100 and -101 resemble the ones of Zeolite Imidazolate Frameworks (ZIFs), ${ }^{[32]}$ which are an important family of MOFs (a subclass of coordination polymers) and are isomorphic with zeolites from topological perspective. Further investigation of the correlation of FPOPs' Young's moduli and densities was done in order to compare with ZIFs and ten other common polymers. Densities $(\rho)$ of FPOPs were measured by using Mettler Toledo kit based on Archimedes' principle. $\rho$ of FPOP-100 $\left(\rho=1440.03 \pm 46.29 \mathrm{~kg} / \mathrm{m}^{3}\right)$ and $-101(\rho=1451.61 \pm$ $20.67 \mathrm{~kg} / \mathrm{m}^{3}$ ) are higher than ZIF-8 (Fig. 6), which has nearly the same $E$ but higher $H(H\{110\}$ of $Z I F-8=531 \pm 28 \mathrm{MPa}){ }^{[26,33]}$ This higher hardness of ZIF-8 indicates its ability to withstand higher load when the projected area of residual impression stays the same for both ZIF-8 and FPOPs. In other words, under the same indentation load, FPOPs suffer more permanent

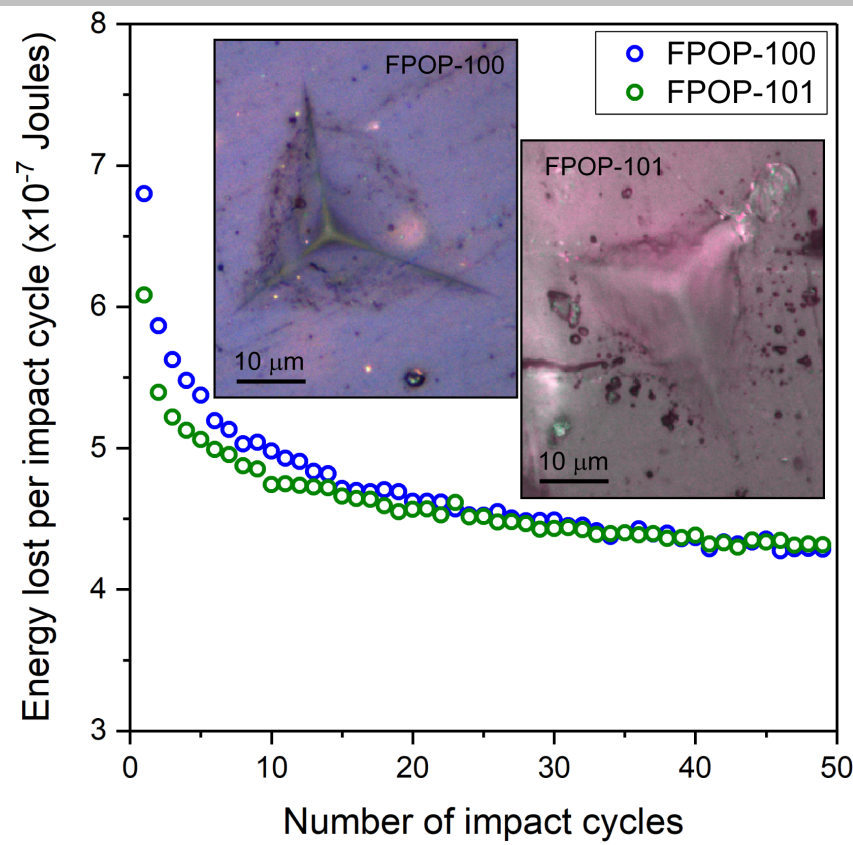

Figure 7. Energy dissipated per impact cycle versus the number of cycles showing the relative toughness of FPOP-100 and FPOP-101. Optical images of the residual impressions on FPOP-100 and FPOP-101 are shown in the insets.

deformation, which could be plastic deformation of the network until the eventual bond breakage.

Using the MTS nanoindenter, we applied cyclic loads to the FPOPs so as to continuously deform the FPOP surfaces. During the cyclic impact study, compaction of the networks occurs, which is reflected by Fig. 7 where the energy lost is reduced over loading cycles. We found that FPOP-100 is tougher than FPOP-101, because the energy of mechanical deformation of FPOP-100 in the first approx. 15 impact cycles is apparently higher than FPOP. 101. Nevertheless, energy lost per impact cycle converges to $4.29 \mathrm{pJ}$ after approx. 40 cycles, and this suggests majority of $\mathrm{CF}_{3}$ groups in both FPOP-100 and $-\mathbf{1 0 1}$ are gradually ruptured leading to the materials' compaction reminiscent of ductile polymers. Further information of the loading conditions is shown in Supporting Information Fig. S33.

Control experiments were important in order to understand the role of fluorines behind inducing hydrophobicity and mechanical stiffness properties. For verification purpose, the substitutions around the precursor dinitriles were fine-tuned: $-\mathrm{CF}_{3}$ groups in $\mathrm{L}^{\prime}$ and $\mathrm{L}^{\prime}$ were replaced by $\mathrm{CH}_{3}$ and $\mathrm{H}$-atoms, making way to three differently substituted dinitrile building blocks ( $L_{1}: 4,4^{\prime}-(3,5-$ dimethylphenylazanediyl)dibenzonitrile; $\quad \mathrm{L}_{2}$ : $\quad 4,4^{\prime}-(2,5-$ dimethylphenylazanediyl)dibenzonitrile; $\quad \mathrm{L}_{3}$ : 4,4'(phenylazanediyl)dibenzonitrile) for making an analogous series of non-fluorinated POPs MPOP-100, MPOP-101, and POP-100 respectively (Figures S9-S17 and S29-S30). As evident from the photographic impressions of these three materials (Figures S29 $a, b$ and $c$ : insets), the physical appearances were those of typical powdered materials devoid of mechanical stiffness, thus unable to form a bulk monolith. Since the other two dintriles $L_{2}$ and $L_{3}$ remained amorphous despite a number of crystallization attempts, crystal packing of $L_{1}$ was considered as a representative of the three non-fluorinated precursors. A closer look at the intermolecular packing modes (evident intermolecular $\mathrm{H}$-bonding and van der Waals interactions) reveal contrasting nature of such interactions (manifold for $L^{\prime}$ and $L^{\prime \prime}$, while merely one-fold for $L_{1}$; considering a single unit cell $(1 \times 1 \times 1)$ for each) (Figures S25 
and S26). Such manifold C-F bond mediated multiple interactions might play key roles to augment the extended crosslinking in the densely packed and fluorinated triazine polymer motifs. To support these analyses from a more practical viewpoint, we measured the static water contact angles (with sessile drops) for all the three non-fluorinated POPs (Fig. S32), and noticed hydrophilic nature for all the three surfaces. When these low WCA values are contrasted against the high superhydrophobic surfacecharacteristics manifested for FPOP-100 and FPOP-101 surfaces, the pivotal role of bis(trifluoromethyl) (-CF $)$-functionalization becomes evident.

\section{Conclusions}

Installation of bis(trifluoromethyl)-functionalization (i.e. $-\mathrm{CF}_{3}$ ) presents an interesting avenue to develop self-assembled, hydrophobic, fluorous porous organic polymers (FPOPs). Mechanical studies have established their stiffness behaviour as analogous to their organic-inorganic hybrid polymer analogues, particularly ZIFs and those of glassy polymers, while concomitant surface hydrophobicity measurements could underpin the $-\mathrm{CF}_{3}$ groups' role behind the observed superhydrophobicity. The present study offers a new paradigm to simultaneously introduce mechanical stiffness and high surface hydrophobicity into polymeric organic networks, and as a novel rationale, may serve as an utilitarian roadmap to guide the engineering of future generation of mechanically stiff and hydrophobic materials.

\section{Experimental Section}

Materials. 4-fluorobenzonitrile (99\%), Cesium fluoride (99\%) and trifluoromethanesulfonic acid (reagent grade, 98\%) were purchased from Sigma-Aldrich. Dry and HPLC grade solvents (including N, Ndimethylformamide) were obtained from Alfa Aesar. 3,5Bis(trifluoromethyl)aniline (>97\%), 2,5-Bis(trifluoromethyl)aniline (>98\%), 3,5-dimethylaniline (>98\%) and 2,5-dimethylaniline (>97\%) were procured from $\mathrm{TCl}$ Chemicals. These chemicals were used without further purification.

Physical measurements. The IR spectra were recorded by using NICOLET 6700 FT-IR spectrophotometer using KBr pellet in $600-3000 \mathrm{~cm}^{-}$ 1 range. Thermogravimetric analysis data were recorded on Perkin-Elmer STA 6000 TGA analyser under $\mathrm{N}_{2}$ atmosphere with heating rate of $10^{\circ} \mathrm{C}$ $\mathrm{min}^{-1}$. Gas adsorption measurements were studied using BelSorp-max instrument from Bel Japan. Contact angles on the FPOP-100 and FPOP101 samples were measured by using a Contact Angle Meter (Model ID: HO-IAD-CAM-01; Holmarc Opto-Mechatronics Pvt. Ltd.), followed by LBADSA drop analysis (ImageJ software), which is based on the fitting of the Young-Laplace equation to the droplet image data (droplet shape analysis), using $10 \mu \mathrm{L}$ distilled water, with an accuracy of $\pm 2^{\circ}$. The morphology of the POP samples are recorded with Zeiss Ultra Plus FESEM. Powder X-ray diffraction (PXRD) data were recorded at room temperature from a Bruker D8 Advance diffractometer, using $\mathrm{Cu} \mathrm{Ka}$ radiation $(\lambda=1.5406 \AA)$. Raman spectra $\left(\lambda_{\text {exc }}=532 \mathrm{~nm}\right)$ were recorded using Raman microscope (LabRAM HR, Horbia Jobin Yvon) with a 60X objective lens. The morphologies of the FPOP nanospheres were characterized using an atomic force microscope (Agilent instrument: Keysight, 5500 AFM). The measurements were performed in a noncontact mode using Si tip, with tip resonance frequency $330 \mathrm{kHz}$ and cantilever thickness $4 \mu \mathrm{m}$. Solid-state Cross-Polarization Magic Angle Spinning Carbon-13 Nuclear Magnetic Resonance ( $\left({ }^{13} \mathrm{C}\right.$ CP-MAS SSNMR) spectra were recorded on a Bruker Advance-III Ultrashield500WB spectrometer (probe: MAS BB 4MM and Spinning rate: $5 \mathrm{KHz}$ ) with guest- free, POP samples (crushed and packed tightly in quartz capillary), whereas liquid state NMR characterization of dinitrile precursors were performed on $400 \mathrm{MHz}$ Jeol ECS-400 (or $100 \mathrm{MHz}$ for ${ }^{13} \mathrm{C}$ ) and $270 \mathrm{MHz}$ JEOL FX-270 NMR (or $67.5 \mathrm{MHz}$ for ${ }^{13} \mathrm{C}$ ) spectrometers. Carbon and proton chemical shifts are expressed in parts per million ( $\delta$ scale).

X-ray Structural Studies. Single-crystal X-ray data of precursors L' and L" were collected at $150 \mathrm{~K}$ on a Bruker D8 Quest diffractometer (operated at $1500 \mathrm{~W}$ power: $50 \mathrm{kV}, 30 \mathrm{~mA}$ ) using graphite-monochromated MoKa radiation $(\lambda=0.71073 \AA$ ). Crystal was mounted on nylon CryoLoops (Hampton Research) with Paraton-N (Hampton Research). The data integration and reduction were processed with SAINT ${ }^{[34]}$ software and Olex $2^{[35]}$ package. A multi-scan absorption correction was applied to the collected reflections. The structure was solved by direct method using SHELXTL ${ }^{[36]}$ and was refined on $F^{2}$ by full-matrix least-squares technique using the SHELXL-97[37] program package within the WINGX[38] programme. All non-hydrogen atoms were anisotropically refined. All hydrogen atoms were located in successive difference Fourier maps and they were treated as riding atoms using SHELXL default parameters. Both the structures were examined using the Adsym subroutine of PLATON ${ }^{[39]}$ to assure that no additional symmetry could be applied to the models. Crystallographic data for L', L" and $L_{1}$ are: CCDC 1823779, 1823780, 1823781, respectively.

Low Pressure Gas and Solvent Sorption measurements. Low pressure gas and solvent sorption measurements were performed using BELSORPmax and BELSORP-aqua3 adsorption analysers (BEL Japan, Inc.) respectively, both equipped with constant temperature bath. All the employed gases were of $99.99 \%$ purity, and the solvents were of HPLC grade. THF/CHCl 3 (1:1)-exchanged phases of the compounds FPOP-100 and FPOP-101 were heated at $80^{\circ} \mathrm{C}$ under vacuum for $2 \mathrm{~h}$, to get guestfree phases of the respective polymers. Prior to each adsorption measurement, the guest-free samples were again pre-treated at $80{ }^{\circ} \mathrm{C}$ under vacuum for $1 \mathrm{~h}$, using BelPrepvacll, and purged with $\mathrm{N}_{2}$ on cooling. All solvent sorption measurements were recorded at $298 \mathrm{~K}$.

Nanoindentation. Nanoindentation experiments were implemented using a commercial system (MTS Nanolndenter ${ }^{\circledR}$ XP), which is a microprobe instrument for measuring mechanical properties. This system is able to measure the interface contact stiffness continuously during each indentation. Berkovich indenter that is a three-sided pyramidal indenter (apex radius $\sim 100 \mathrm{~nm}$ ) was adopted in the experiment to measure Young's modulus and hardness. Additionally, it can also be used to generate cracks for measuring fracture toughness because it is relatively sharper than spherical indenter, ${ }^{[40]}$ viz. smaller tip apex radius, which is apt to create stable elastic-plastic transition rather than inducing cracks. An array of instrumented indentations of ca. $2 \mu \mathrm{m}$ depth has been implemented on both FPOP-100 and FPOP-101, which is mounted on epoxy resin and then polished by polycrystalline diamond suspension (size of the diamond crystals is $\sim 0.1 \mu \mathrm{m}$ in diameter), to achieve efficient cutting and minimal deformation.

\section{Acknowledgements}

IISER Pune is acknowledged for providing research fellowship and facilities, while SERB India (Project No. EMR/2016/000410) is acknowledged for generous financial support. P.S. thanks UGC for senior research fellowship. Z.Z. is grateful to Prof. John Huber and Prof. Steve Roberts for kindly allowing us the access to AFM instrumentation and MTS Nanoindenter, respectively.

Keywords: Porous organic polymer • Mechanical stiffness • Hydrophobicity • Fluorine • 
[1] a) J.-X. Jiang, A. I. Cooper, in Functional Metal-Organic Frameworks: Gas Storage, Separation and Catalysis (Ed.: M. Schröder), Springer Berlin Heidelberg, Berlin, Heidelberg, 2010, pp. 1-33; b) Y. Xu, S. Jin, H Xu, A. Nagai, D. Jiang, Chem. Soc. Rev. 2013, 42, 8012-8031; c) D. Wu, F. Xu, B. Sun, R. Fu, H. He, K. Matyjaszewski, Chem. Rev. 2012, 112 3959-4015; d) E. Merino, E. Verde-Sesto, E. M. Maya, M. Iglesias, F Sánchez, A. Corma, Chem. Mater. 2013, 25, 981-988; e) P. Samanta, P. Chandra, A. V. Desai, S. K. Ghosh, Mater. Chem. Front. 2017, 1, 1384 1388.

[2] a) C. R. DeBlase, K. E. Silberstein, T.-T. Truong, H. D. Abruña, W. R. Dichtel, J. Am. Chem. Soc. 2013, 135, 16821-16824; b) J. C. Tan, A. K Cheetham, Chem. Soc. Rev. 2011, 40, 1059-1080.

[3] a) H. Xu, J. Gao, D. Jiang, Nat Chem 2015, 7, 905-912; b) Y. Peng, Z. Hu, Y. Gao, D. Yuan, Z. Kang, Y. Qian, N. Yan, D. Zhao, ChemSusChem 2015, 8, 3208-3212; c) M. H. Alkordi, L. J. Weselinski, V. D'Elia, S. Barman, A. Cadiau, M. N. Hedhili, A. J. Cairns, R. G. AbdulHalim, J.-M. Basset, M. Eddaoudi, J. Mater. Chem. A 2016, 4, 7453-7460.

[4] a) S. Wan, J. Guo, J. Kim, H. Ihee, D. Jiang, Angew. Chem. Int. Ed. 2008, 47, 8826-8830; b) S. Dalapati, E. Jin, M. Addicoat, T. Heine, D. Jiang, J. Am. Chem. Soc. 2016, 138, 5797-5800; c) D. Gopalakrishnan, W. R. Dichtel, J. Am. Chem. Soc. 2013, 135, 8357-8362; d) J. Dong, K. Zhang X. Li, Y. Qian, H. Zhu, D. Yuan, Q.-H. Xu, J. Jiang, D. Zhao, Nat Commun. 2017, 8, 1142.

[5] a) A. Karmakar, A. Kumar, A. K. Chaudhari, P. Samanta, A. V. Desai, R. Krishna, S. K. Ghosh, Chem. Eur. J. 2016, 22, 4931-4937; b) C. J. Doonan, D. J. Tranchemontagne, T. G. Glover, J. R. Hunt, O. M. Yaghi, Nat Chem 2010, 2, 235-238; c) S. Hug, L. Stegbauer, H. Oh, M. Hirscher, B. V. Lotsch, Chem. Mater. 2015, 27, 8001-8010.

[6] a) H. Takeda, M. Ohashi, Y. Goto, T. Ohsuna, T. Tani, S. Inagaki, Chem. Eur. J. 2014, 20, 9130-9136; b) K. Schwinghammer, S. Hug, M. B. Mesch J. Senker, B. V. Lotsch, Energy Environ. Sci. 2015, 8, 3345-3353; c) V. S. Vyas, F. Haase, L. Stegbauer, G. Savasci, F. Podjaski, C. Ochsenfeld B. V. Lotsch, Nat Commun 2015, 6.

[7] a) Q. Fang, J. Wang, S. Gu, R. B. Kaspar, Z. Zhuang, J. Zheng, H. Guo, S. Qiu, Y. Yan, J. Am. Chem. Soc. 2015, 137, 8352-8355; b) L. Bai, S. Z. F. Phua, W. Q. Lim, A. Jana, Z. Luo, H. P. Tham, L. Zhao, Q. Gao, Y. Zhao, Chem. Commun. 2016, 52, 4128-4131.

[8] a) T. Uemura, T. Kaseda, Y. Sasaki, M. Inukai, T. Toriyama, A. Takahara, H. Jinnai, S. Kitagawa, Nat Commun 2015, 6; b) P. J. Waller, F. Gándara, O. M. Yaghi, Accounts of Chemical Research 2015, 48, 3053-3063; c) Y.-B. Huang, P. Pachfule, J.-K. Sun, Q. Xu, J. Mater. Chem. A 2016, 4, 4273-4279.

[9] a) C. S. Diercks, O. M. Yaghi, Science 2017, 355; b) S. Wan, J. Guo, J. Kim, H. Ihee, D. Jiang, Angew. Chem. Int. Ed. 2009, 48, 5439-5442; c) P. Samanta, A. V. Desai, B. Anothumakkool, M. M. Shirolkar, A. Karmakar, S. Kurungot, S. K. Ghosh, J. Mater. Chem. A 2017, 5, 13659 13664.

[10] Q. Sun, B. Aguila, J. Perman, L. D. Earl, C. W. Abney, Y. Cheng, H. Wei, N. Nguyen, L. Wojtas, S. Ma, J. Am. Chem. Soc. 2017, 139, 2786-2793.

[11] K. Wang, L. M. Yang, X. Wang, L. Guo, G. Cheng, C. Zhang, S. Jin, B. Tan, A. Cooper, Angew. Chem. Int. Ed. 2017, 56, 14149-14153.

[12] a) P. Kuhn, M. Antonietti, A. Thomas, Angew. Chem. Int. Ed. 2008, 47, 3450-3453; b) Y. Zhao, K. X. Yao, B. Teng, T. Zhang, Y. Han, Energy Environ. Sci. 2013, 6, 3684-3692; c) S. Kuecken, J. Schmidt, L. Zhi, A. Thomas, J. Mater. Chem. A 2015, 3, 24422-24427.

[13] a) S. Ren, M. J. Bojdys, R. Dawson, A. Laybourn, Y. Z. Khimyak, D. J. Adams, A. I. Cooper, Adv. Mater. 2012, 24, 2357-2361; b) X. Zhu, C Tian, S. M. Mahurin, S.-H. Chai, C. Wang, S. Brown, G. M. Veith, H. Luo, H. Liu, S. Dai, J. Am. Chem. Soc. 2012, 134, 10478-10484; c) C. E. Chan-Thaw, A. Villa, P. Katekomol, D. Su, A. Thomas, L. Prati, Nano Lett 2010, 10, 537-541; d) L. Hao, J. Ning, B. Luo, B. Wang, Y. Zhang, Z. Tang, J. Yang, A. Thomas, L. Zhi, J. Am. Chem. Soc. 2015, 137, 219225; e) R. Palkovits, M. Antonietti, P. Kuhn, A. Thomas, F. Schüth Angew. Chem. Int. Ed. 2009, 48, 6909-6912; f) H. Liao, H. Ding, B. Li, X Ai, C. Wang, J. Mater. Chem. A 2014, 2, 8854-8858.

[14] a) Z.-F. Pang, S.-Q. Xu, T.-Y. Zhou, R.-R. Liang, T.-G. Zhan, X. Zhao, J. Am. Chem. Soc. 2016, 138, 4710-4713; b) M. Tong, Q. Yang, Y. Xiao, C Zhong, Phys. Chem. Chem. Phys. 2014, 16, 15189-15198; c) Y. Zeng, R. Zou, Y. Zhao, Adv. Mater. 2016, 28, 2855-2873.
[15] a) S. Mukherjee, A. M. Kansara, D. Saha, R. Gonnade, D. Mullangi, B. Manna, A. V. Desai, S. H. Thorat, P. S. Singh, A. Mukherjee, S. K. Ghosh, Chem. Eur. J. 2016, 22, 10937-10943; b) T.-H. Chen, I. Popov, O Zenasni, O. Daugulis, O. S. Miljanic, Chem. Commun. 2013, 49, 68466848

[16] D. F. Bahr, J. A. Reid, W. M. Mook, C. A. Bauer, R. Stumpf, A. J. Skulan N. R. Moody, B. A. Simmons, M. M. Shindel, M. D. Allendorf, Phys. Rev. B 2007, 76, 184106

[17] a) H. Ma, H. Ren, S. Meng, F. Sun, G. Zhu, Sci. Rep. 2013, 3, 2611; b) L.-M. Tao, F. Niu, D. Zhang, T.-M. Wang, Q.-H. Wang, New J. Chem 2014, 38, 2774-2777; c) F. Niu, L. Tao, Y. Deng, H. Gao, J. Liu, W. Song, New J. Chem. 2014, 38, 5695-5699; d) K. Sakaushi, G. Nickerl, F. M. Wisser, D. Nishio-Hamane, E. Hosono, H. Zhou, S. Kaskel, J. Eckert, Angew. Chem. Int. Ed. 2012, 51, 7850-7854.

[18] X. Feng, X. Ding, D. Jiang, Chem. Soc. Rev. 2012, 41, 6010-6022.

[19] S. Yuan, B. Dorney, D. White, S. Kirklin, P. Zapol, L. Yu, D.-J. Liu, Chem. Commun. 2010, 46, 4547-4549.

[20] V. A. Davankov, M. P. Tsyurupa, Reactive Polymers 1990, 13, 27-42.

[21] P. M. Budd, B. S. Ghanem, S. Makhseed, N. B. McKeown, K. J. Msayib, C. E. Tattershall, Chem. Commun. 2004, 230-231.

[22] T. Ben, H. Ren, S. Ma, D. Cao, J. Lan, X. Jing, W. Wang, J. Xu, F. Deng, J. M. Simmons, S. Quu, G. Zhu, Angew. Chem. Int. Ed. 2009, 48, 94579460.

[23] a) J. X. Jiang, F. Su, A. Trewin, C. D. Wood, N. L. Campbell, H. Niu, C. Dickinson, A. Y. Ganin, M. J. Rosseinsky, Y. Z. Khimyak, A. I. Cooper, Angew. Chem. Int. Ed. 2007, 46, 8574-8578; b) A. I. Cooper, Adv. Mater. 2009, 21, 1291-1295.

[24] R. Dawson, A. I. Cooper, D. J. Adams, Prog. Polym. Sci. 2012, 37, 530563.

[25] a) Z.-R. Jiang, J. Ge, Y.-X. Zhou, Z. U. Wang, D. Chen, S.-H. Yu, H.-L. Jiang, NPG Asia Mater. 2016, 8, e253; b) D. Mullangi, S. Shalini, S. Nandi, B. Choksi, R. Vaidhyanathan, J. Mater. Chem. A 2017, 5, 83768384

[26] J. C. Tan, T. D. Bennett, A. K. Cheetham, Proc. Natl. Acad. Sci. U.S.A. 2010, 107, 9938-9943.

[27] P. Puthiaraj, Y.-R. Lee, S. Zhang, W.-S. Ahn, J. Mater. Chem. A 2016, 4, 16288-16311.

[28] a) K. Sakaushi, E. Hosono, G. Nickerl, H. Zhou, S. Kaskel, J. Eckert, J. Power Sources 2014, 245, 553-556; b) T. Zheng, W. Xing, J. R. Dahn Carbon 1996, 34, 1501-1507

[29] S.-i. Noro, T. Nakamura, NPG Asia Mater. 2017, 9, e433.

[30] C. Yang, U. Kaipa, Q. Z. Mather, X. Wang, V. Nesterov, A. F. Venero, M. A. Omary, J. Am. Chem. Soc. 2011, 133, 18094-18097.

[31] D.-P. Liu, Q. Chen, Y.-C. Zhao, L.-M. Zhang, A.-D. Qi, B.-H. Han, ACS Macro Lett. 2013, 2, 522-526.

[32] a) R. Banerjee, A. Phan, B. Wang, C. Knobler, H. Furukawa, M. O'Keeffe, O. M. Yaghi, Science 2008, 319, 939-943; b) S. El-Hankari, J. AguileraSigalat, D. Bradshaw, J. Mater. Chem. A 2016, 4, 13509-13518.

[33] Z. Zeng, J.-C. Tan, ACS Appl. Mater. Interfaces 2017, 9, 39839-39854.

[34] SAINT Plus, (Version 7.03); Bruker AXS Inc.: Madison, WI, 2004.

[35] O. V. Dolomanov, L. J. Bourhis, R. J. Gildea, J. A. K. Howard, H. Puschmann, J. Appl. Crystallogr. 2009, 42, 339-341.

[36] Sheldrick, G. M. SHELXTL, Reference Manual: version 5.1: Bruker AXS; Madison, WI, 1997.

[37] G. Sheldrick, Acta Crystallographica Section A 2008, 64, 112-122.

[38] WINGX version 1.80.05 Louis Farrugia, University of Glasgow.

[39] Spek, A. L. PLATON, A Multipurpose Crystallographic Tool, Utrecht University, Utrecht, The Netherlands, 2005.

[40] J. C. Tan, C. A. Merrill, J. B. Orton, A. K. Cheetham, Acta Mater. 2009 57, 3481-3496. 


\section{Entry for the Table of Contents}

\section{FULL PAPER}

As a prototypal design strategy in the porous materials' regime, specific fluorine-rich substituents could infuse mechanical stiffness and high surface hydrophobicity properties among a pair of functionalized porous organic polymers.

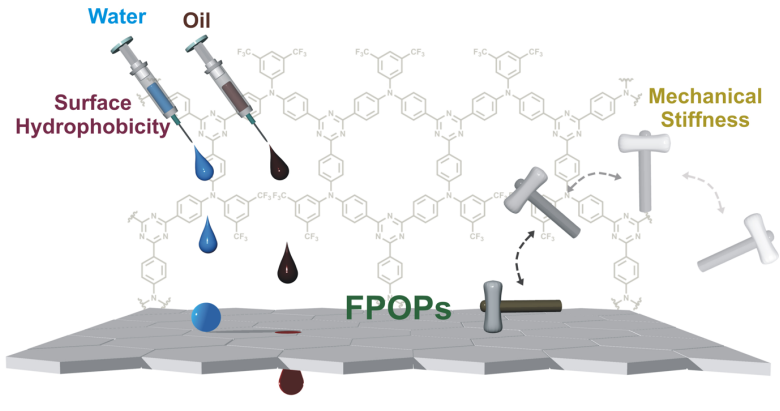

Soumya Mukherjee, Zhixin Zeng, Mandar M. Shirolkar, Partha Samanta, Abhijeet $K$. Chaudhari, Jin-Chong Tan, and Sujit K. Ghosh*

Page No. - Page No.

Self-assembled, Fluorinerich Porous Organic Polymers: A Class of Mechanically Stiff and Hydrophobic Materials 